\title{
(J) Increase in sensory sensitivity around the hyperkeratotic papule may be involved in pruritus in lichen amyloidosis
}

\author{
Tsugunobu Andoh ${ }^{1}$ and Tadamichi Shimizu ${ }^{2}$
}

${ }^{1}$ Department of Applied Pharmacology, ${ }^{2}$ Department of Dermatology, Graduate School of Medicine and Pharmaceutical Sciences, University of Toyama, Japan

\section{Aim of this study}

Lichen amyloidosis (LA) is an itchy skin disorder characterized by amyloid deposits in the superficial dermis. Pruritus is the presenting symptom in $90 \%$ of LA patients. However, the underlying mechanisms and mediators of pruritus in LA remain unclear.

One LA patient complained of severe pruritus in the hyperkeratotic papules, especially around the papule. In pruritic skin diseases, skin sensitivity is increased against tactile stimulation. Thus, in this study, we evaluated in detail the sensitivity against tactile stimulation in the skin, especially the hyperkeratotic papule

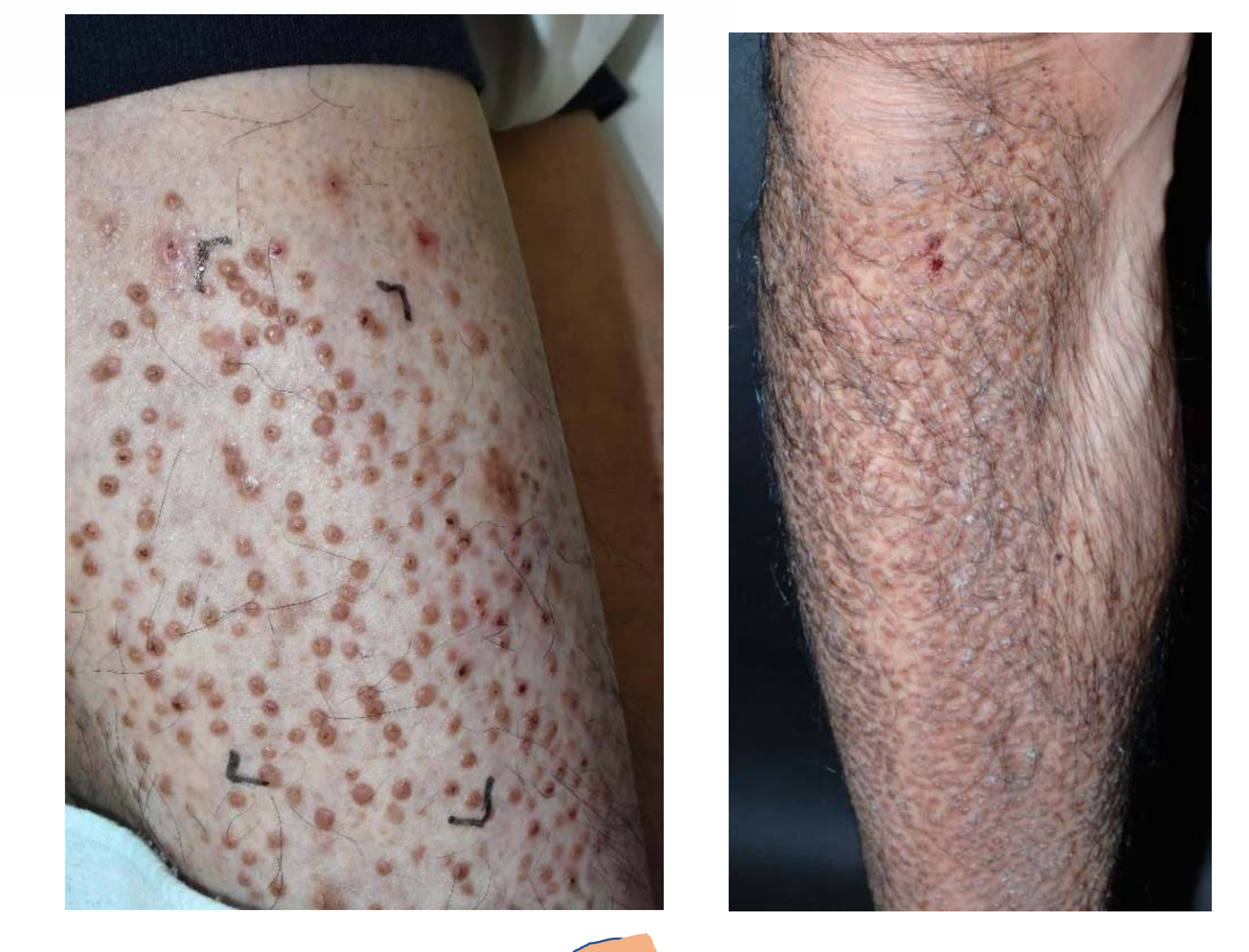
Itch

Mechanisms? and its edge, of LA patients.

\section{Materials \& Methods}

\section{$>$ Subjects}

Six patients with LA (six men; mean age, 47 years; range, 27-77 years) enrolled at Toyama University Hospital and five healthy subjects (Two women and three men; mean age, 50 years; range 34-65 years) employed at the University of Toyama were underwent touch test. Skin biopsy was performed in six patients with LA (two women and four men; mean age, 51 years; range 23-77 years) enrolled at Toyama University Hospital. This study was approved by the Medical Ethics Committee of the University of Toyama, Toyama, Japan.

\section{Touch test}

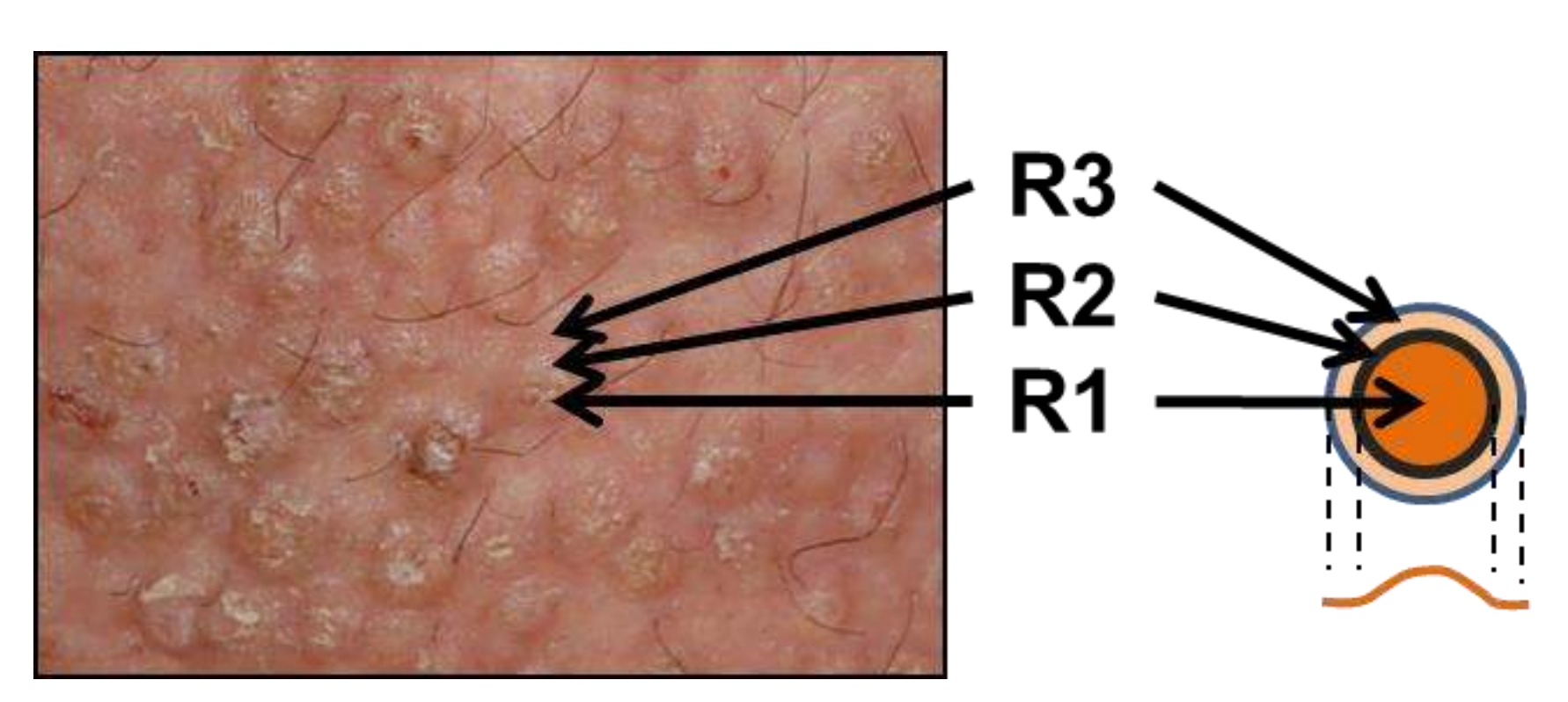

Non-noxious mechanical stimulation was given to patients with LA using a fine von Frey filament (North Coast Medical Inc). A von Frey filament with a bending force of $0.69 \mathrm{mN}$ was applied perpendicularly against the central part of each point of the hyperkeratotic papule or its periphery: R1, center of the hyperkeratotic papule; R2, edge of the hyperkeratotic papule; R3, approximately $1-0.5 \mathrm{~mm}$ from the edge of the hyperkeratotic papule; R0, healthy skin in patients with cutaneous amyloidosis. Responses to the stimuli were scored as follows: $\mathbf{0}$, patient did not feel the stimulus; 1 , patient felt the stimulus. The test was performed in six hyperkeratotic papules or healthy skin per patient with cutaneous amyloidosis.

\section{Immunohistochamical analysis}

Skin samples were fixed in $10 \%$ neutral buffered and then embedded in paraffin. The paraffin blocks were cut with a microtome and the sections were placed on standard slides. After deparaffinization, these sections were stained Dylon or toluidine blue. For immunohistochemical stain, the sections were reacted with several antibodies (e.g. anti-protein gene product 9.5 (Ultraclone), anti-nerve growth factor (Merck), or anti-5lipoxygenase antibody (Santa Cruz Biotechnology)). And then these sections were reacted with horseradish peroxidase-conjugated avidin (DAKO) following by reacting with biotin-conjugated anti-IgG antibody (DAKO). The sections were subsequently treated with 3,3'-diaminobenzidine tetrahydrochloride (DAKO) and counterstained with hematoxylin. The preparations were mounted with Canada balsam and observed using a light microscope (Olympus) with a CCD camera.

\section{Results}

\section{Cutaneous sensory sensitivity hyperkeratotic papule of}

\section{lichen amyloidosis}

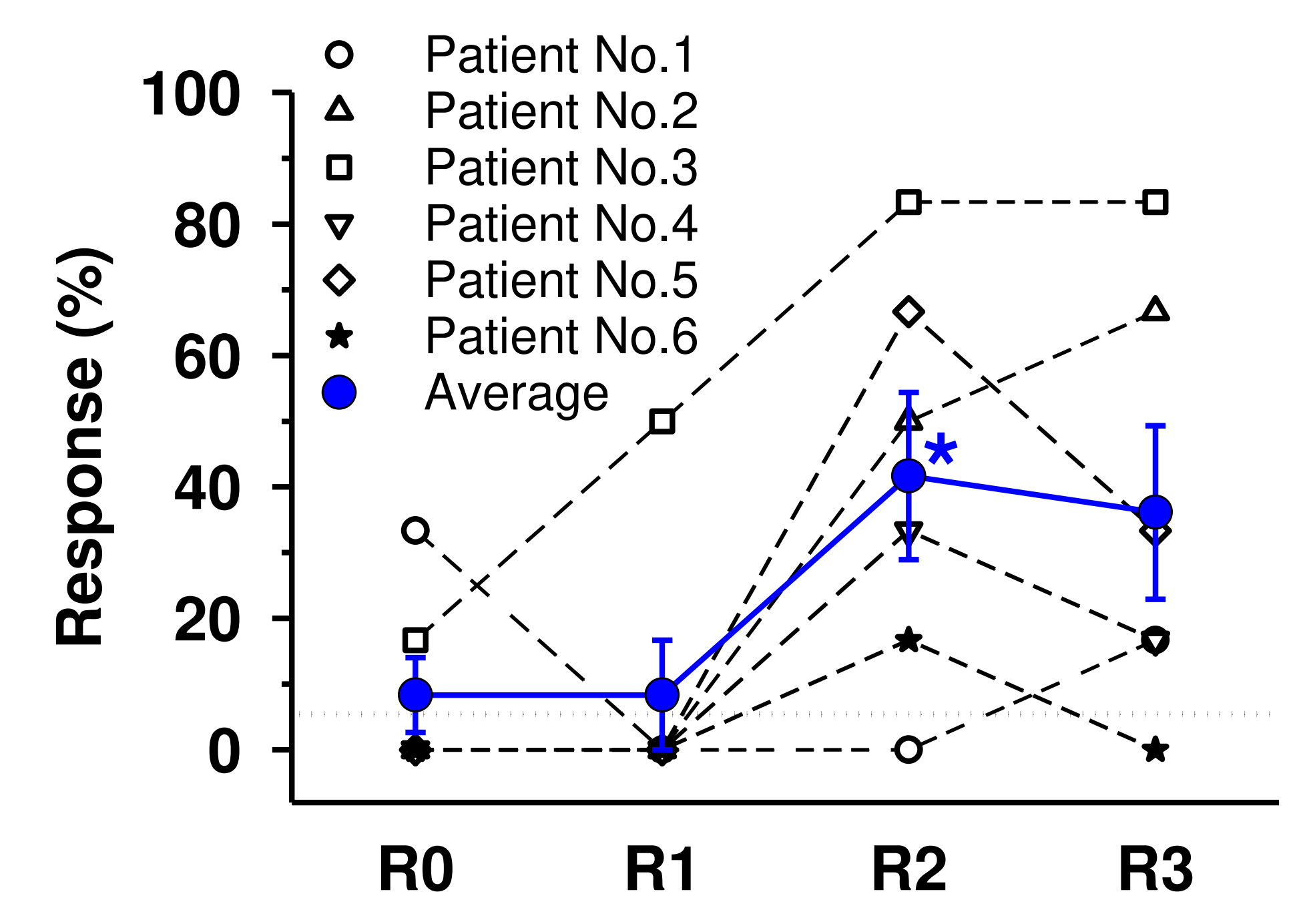

In the center of the hyperkeratotic papules, the patients' response against von Frey filament was low. However, when the edge of the hyperkeratotic papule was stimulated with von Frey filament, the LA patients responded highly. In addition, the LA patients also responded against the stimulation of the periphery of the hyperkeratotic papule with von Frey filament ( $P=0.0784)$.

\section{Typical examples of histochemical staining in skin with}

lichen amyloidosis
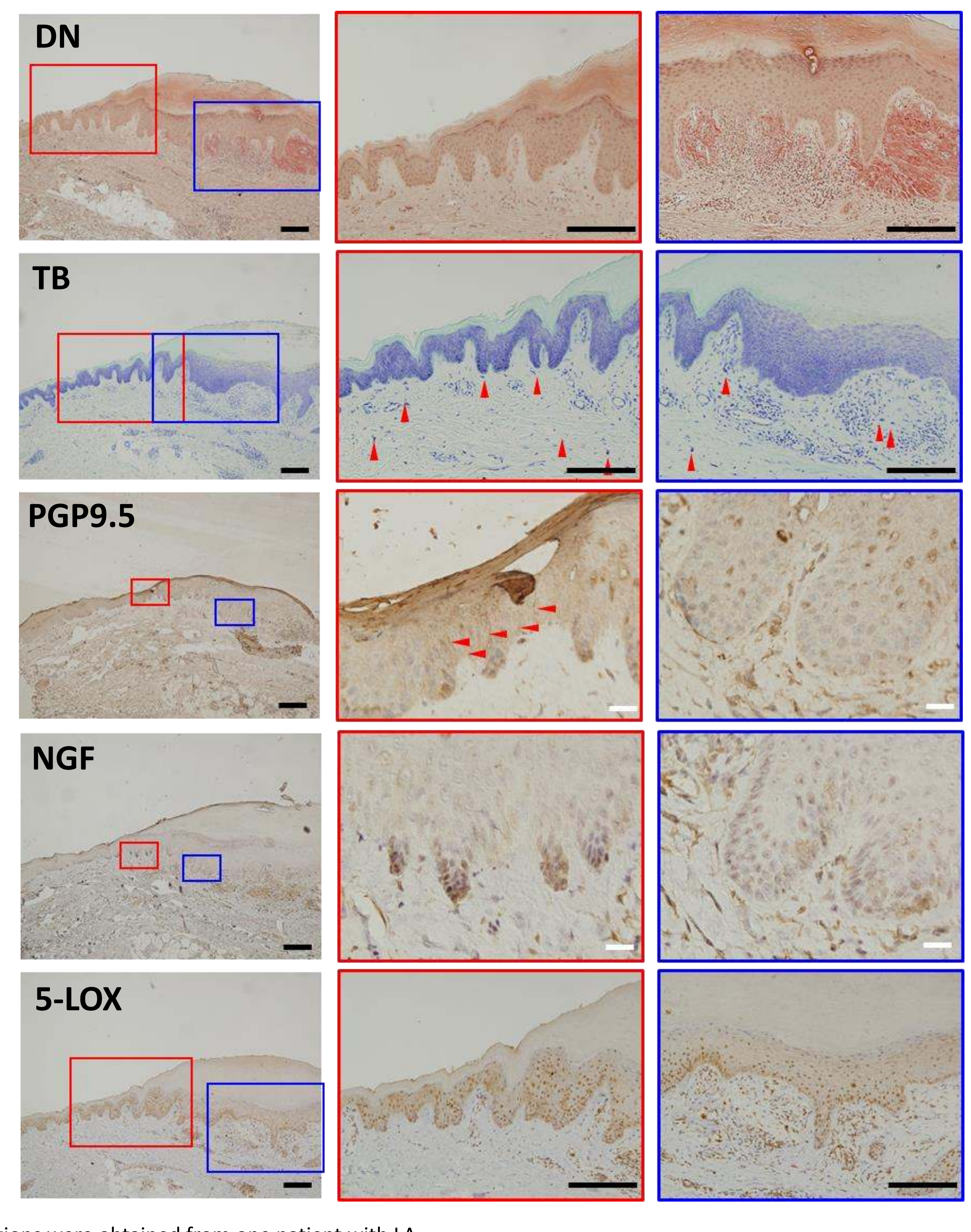

DN, Dyron stain; TB, toluidine

(arow head: primary sensory neuron), nerve growth factor (NGF), and 5-lipoxygenase (5-LOX).

a Scale bar: black bar, $200 \mu \mathrm{m}$; white bar, $20 \mu \mathrm{m}$

- [DN stain] Amyloid deposition was observed in the papillary dermis under the hyperkeratotic papules, but not around them.

- [TB stain] A small number of mast cells were distributed widely in the dermis.

- [PGP9.5 immunoreactivity] The immunoreactivity of PGP9.5, which is a marker of neuron, was strongly observed on the epidermis of the edge of the hyperkeratotic papules, but not under the hyperkeratotic papules.

- [NGF immunoreactivity] The NGF-immunoreactivity at the edge, especially the epidermal ridges, of the hyperkeratotic papules was observed. However, the immunoreactivity was not detected under the hyperkeratotic papules.

- [5-LOX immunoreactivity] High 5-LOX immunoreactivity was detected around the hyperkeratotic papules, especially at their edges, in LA patients. However, under the hyperkeratotic papules, there was little immunoreactivity.

\section{PGP 9.5-, NGF- and 5-LOX-immunoreactivity in each patients} with lichen amyloidosis

\begin{tabular}{|c|ccc|c|c|c|c|c|c|}
\hline \multirow{2}{*}{ Patients } & \multicolumn{3}{|c|}{ PGP9.5 } & \multicolumn{3}{|c|}{ NGF } & \multicolumn{3}{|c|}{ 5-LOX } \\
\cline { 2 - 10 } No1 & R1 & R2 & R3 & R1 & R2 & R3 & R1 & R2 & R3 \\
\hline No2 & - & + & n.d. & - & + & - & - & ++ & n.d. \\
\hline No3 & + & ++ & ++ & - & + & - & + & ++ & ++ \\
\hline No4 & + & ++ & ++ & - & + & - & + & + & ++ \\
\hline No5 & - & + & - & - & + & + & + & ++ & n.d. \\
\hline No6 & + & + & + & - & + & + & + & + & + \\
\hline
\end{tabular}

$>$ n.d., no area in the section, Immunorecativity: - , no or little stained; + , stained; ++ , highly stained.

PGP9.5, protein gene product 9.5; NGF, nerve growth factor; 5-LOX, 5-lipoxygenase

\section{A schematic conclusion}

In summary, we demonstrated that the sensory sensitivity at the edge, but not at the center, of hyperkeratotic papules in LA patients was increased through the epidermal elongation of peripheral sensory neurons. Moreover, we also found an increase in 5-LOX expression, which was related to the production of a potent itch mediator leukotriene $B_{4}\left(L^{2} B_{4}\right)$. Taken together, it is suggested that, at the edge of hyperkeratotic papules in LA patients, the epidermal elongation of peripheral sensory neurons and the production of itch mediators (e.g. $\mathrm{LTB}_{4}$ ) are involved in itch in LA patients. 